\title{
Reply to: Prognostic implications of vitamin $D$ in patients with COVID-19
}

\author{
Claire E. Hastie ${ }^{1} \cdot$ Jill P. Pell $^{1} \cdot$ Naveed Sattar $^{2}$ \\ Published online: 23 November 2020 \\ c) Springer-Verlag GmbH Germany, part of Springer Nature 2020
}

We thank Professor Missouris and colleagues for their interest in our paper. We agree that more data are needed; however, measuring 25-hydroxyvitamin $\mathrm{D}(25(\mathrm{OH}) \mathrm{D})$ concentration in people who are already infected with SARSCoV-2 will suffer from reverse causality as circulating vitamin $\mathrm{D}$ exhibits negative acute phase reactant behavior $[1,2]$. All observational data have limitations and, despite the acknowledged limitations of using UK Biobank for this research question, the work we published perhaps remains as powerful as any to date. This is given key strengths of having access to serum $25(\mathrm{OH}) \mathrm{D}$ concentration measured before infection, its large scale, and adjustment for factors known to strongly influence vitamin D (i.e., ethnicity, body mass index, age, comorbidities, and socioeconomic deprivation). Many other studies that have not considered all relevant factors may have reported positive associations, but with a high likelihood of residual confounding.

The only way to get to the truth of whether vitamin D status matters to COVID-19 outcomes is a randomised controlled trial (RCT). One should not assume that vitamin D supplementation will lessen risk of severe outcome since there is no evidence that such supplementation lessens risk of chronic diseases (e.g., type 2 diabetes, heart failure, renal disease) shown to be associated with increased likelihood of severe outcomes. Of course, whether vitamin D supplementation lessens infection risk is a separate question, and again only robustly conducted RCTs will provide such answers. We are aware of some ongoing randomised trials, e.g., [3] and judgement should await the results of these.

We fully agree that vitamin D supplementation in accordance to the existing national guidelines should be recommended to the general public. Although we would be delighted if vitamin D supplementation did lessen risk of infection or severe COVID-19, there are currently no robust trials addressing this question.

\section{References}

1. Reid D, Knox S, Talwar D, O'Reilly DJ, Blackwell S, Kinsella J et al (2010) Acute changes in the systemic inflammatory response is associated with transient decreases in circulating 25-hydroxyvitamin D concentrations following elective knee arthoplasty. Ann Clin Biochem 47(Suppl 1):95-96

2. Louw JA, Werbeck A, Louw ME, Kotze TJ, Cooper R, Labadarios D (1992) Blood vitamin concentrations during the acute-phase response. Crit Care Med 20(934-41):3

3. https://clinicaltrials.gov/ct2/show/NCT04344041

This reply refers to a letter available here:doi:https://doi. org/10.1007/s00394-020-02429-4.

This reply refers to the original publication available here:doi:https ://doi.org/10.1007/s00394-020-02372-4.

Claire E. Hastie

claire.hastie@glasgow.ac.uk

1 Institute of Health and Wellbeing, University of Glasgow, 1 Lilybank Gardens, Glasgow G12 8RZ, UK

2 Institute of Cardiovascular and Medical Sciences, University of Glasgow, Glasgow, UK 